\title{
Low-frequency low energy ultrasound combined with microbubbles induces distinct apoptosis of A7r5 cells
}

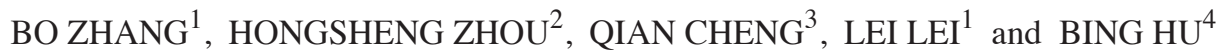 \\ ${ }^{1}$ Department of Echocardiography, Tongji University Affiliated Shanghai East Hospital, Shanghai 200120; \\ ${ }^{2}$ Shanghai Acoustics Laboratory, Chinese Academy of Sciences, Shanghai 200233; ${ }^{3}$ Institute of Acoustics, \\ Tongji University, Shanghai 200092; ${ }^{4}$ Department of Ultrasound in Medicine, Shanghai Institute of Ultrasound \\ in Medicine, Shanghai Jiao Tong University Affiliated 6th People's Hospital, Shanghai 200233, P.R. China
}

Received October 28, 2013; Accepted May 28, 2014

DOI: $10.3892 / \mathrm{mmr} .2014 .2654$

\begin{abstract}
The present study aimed to investigate whether low frequency low energy ultrasound combined with microbubbles induces apoptotic cell death of A7r5 rat aortic vascular smooth muscle cells, and to identify the possible mechanisms underlying this effect. Ultrasonic waves $\left(45 \mathrm{kHz}\right.$ with $0.3 \mathrm{Wcm}^{2}$ of intensity for $0,10,20$ and $30 \mathrm{sec}$ ) were used together with different dosages of SonoVue ${ }^{\mathrm{TM}}$ microbubbles $(0,14,28,42$ and $56 \mu 1$ ), respectively. The cell viability and apoptotic rate were determined by trypan blue staining immediately following treatment and flow cytometry $24 \mathrm{~h}$ thereafter. The treatment conditions resulting in the lowest amount of necrosis, highest apoptotic rate and lowest microbubble dosage was selected for the US+MB group, which was treated with ultrasound combined with microbubbles. The cell proliferation $24 \mathrm{~h}$ following treatment was determined and western blot analysis was applied to examine the expression of apoptosis-associated proteins, B-cell lymphoma 2 (Bcl-2) and Bcl-2-associated $\mathrm{X}$ (Bax). The harmonic acoustic pressure amplitude was measured to obtain the cavitation intensity. The combination of $20 \mathrm{sec}$ ultrasound irradiation and $14 \mu \mathrm{l}$ SonoVue $^{\mathrm{TM}}$ was selected as the treatment conditions for the US+MB group. The results demonstrated that both ultrasound alone (the US group) and in combination with microbubbles significantly inhibited the proliferation of A7r5 cells compared with that of the control $(\mathrm{P}<0.01)$, and the suppression in the US+MB group was significantly greater $(\mathrm{P}<0.01)$. The apoptotic rate in $\mathrm{A} 7 \mathrm{r} 5$ cells induced by this combination treatment $(16.62 \pm 0.93 \%)$ was significantly higher than that in the control $(3.93 \pm 0.39 \%$; $\mathrm{P}<0.01)$ and US $(6.88 \pm 1.87 \% ; \mathrm{P}<0.01)$ groups. Treatment with
\end{abstract}

Correspondence to: Professor Bing Hu, Department of Ultrasound in Medicine, Shanghai Institute of Ultrasound in Medicine, Shanghai Jiao Tong University Affiliated 6th People's Hospital, 600 Yishan Road, Shanghai 200233, P.R. China

E-mail: binghubo@aliyun.com

Key words: low frequency low energy ultrasound, microbubbles, A7r5 cells, apoptosis, Bcl-2, Bax ultrasound combined with microbubbles increased the expression of Bax and decreased the ratio of $\mathrm{Bcl}-2 / \mathrm{Bax}$ compared with those in the control and US groups. The cavitation induced by ultrasound combined with microbubble treatment was more intense than that by ultrasound alone. The results demonstrated that the cell death and apoptosis of A7r5 cells were associated with ultrasound duration and microbubble dosage. Low frequency ultrasound combined with microbubbles induced apoptosis in A7r5 cells through the upregulation of Bax and the downregulation of the Bcl-2/Bax ratio, where the cavitation effect may have an important role.

\section{Introduction}

Restenosis of blood vessels following percutaneous transluminal angioplasty (PTA) and stenting has become a key clinical problem in long-term curative effects, and the proliferation of vascular smooth muscle cells (VSMCs) has an important role in this pathological process (1). Currently, endovascular radiotherapy and gene therapy are the main preventive treatments. However, these methods are not able to completely solve the problem of restenosis. Therefore, it is important to find a simple, safe and effective method to induce apoptosis and inhibit proliferation of VSMCs in the prevention of restenosis.

Ultrasound is a type of non-ionizing energy, which has a number of mechanical, thermodynamical and chemical biological effects. Ultrasound may damage cells irreversibly and induce severe cell membrane modifications (2-4). The effect of ultrasound on cells has been correlated with the generation of acoustic cavitations $(2,5,6)$. Several studies have suggested that cavitations resulting from the collapse of gas bubbles generated by acoustic pressure fields may be the cause of cell damage following ultrasonic irradiation $(7,8)$. Numerous studies have demonstrated that ultrasound is able to induce the apoptosis of endothelial cells and several tumor cells $(9,10)$. However, there have been few reports regarding the effect of ultrasound on VSMCs and there are limited studies on the effect of ultrasound on restenosis (11).

In recent years, the use of low-frequency $(10-60 \mathrm{kHz})$ ultrasound for the enhancement of various biotechnological processes has received increasing attention as a rapid and reagentless method (12). Lower frequency acoustic waves 
have greater tissue penetration than higher frequency acoustic waves due to their longer wavelength, therefore they are particularly suited for transmission into deeper tissue. It has been proven that $20 \mathrm{kHz}$ ultrasound has antitumor effects by inhibiting tumor cell proliferation and inducing apoptosis (13). It has been demonstrated that ultrasound irradiation at $45 \mathrm{kHz}$ and $25 \mathrm{mWcm}^{2}$ induced proliferation and differentiation of odontoblast-like cells (14). It is hypothesized that lower frequency ultrasound may have cavitational effects on VSMCs and affect VSMC proliferation and apoptosis, which may potentially be harnessed as a therapy for preventing restenosis of blood vessels following PTA. However, to date, there have been few studies on the effect of irradiation of low-frequency low-energy ultrasound $(10-60 \mathrm{kHz})$ combined with bubble cavitation on apoptosis of VSMCs (11), and the therapeutic use of low-frequency kilohertz ultrasound for preventing restenosis of blood vessels following PTA and stenting has not been well characterized.

The present study aimed to examine the effect and investigate the possibility of low-frequency low-energy ultrasound combined with microbubbles in preventing restenosis following PTA and stenting. To the best of our knowledge, this is the first study investigating the effects of ultrasound combined with microbubbles treatment on apoptosis and apoptosis-associated proteins of VSMCs.

\section{Materials and methods}

Ethical Consideration. The present study was approved by the ethics committee of Shanghai Jiao Tong University Affiliated 6th People's Hospital, Shanghai Institute of Ultrasound in Medicine (Shanghai, China).

Cell preparation. A7r5 cells were purchased from the Cell Bank of the Chinese Academy of Science Cells (Shanghai, China) and were cultured in $25-\mathrm{cm}^{2}$ culture flasks (Corning Inc., Corning, New York, USA). The cells were grown in Dulbecco's modified Eagle's medium (DMEM) containing $10 \%$ fetal bovine serum (Invitrogen Life Technologies, Paisley, UK), $1 \%$ penicillin and $1 \%$ streptomycin (Sigma-Aldrich, St. Louis, MO, USA), and were maintained at $37^{\circ} \mathrm{C}$ and $5 \%$ $\mathrm{CO}_{2}$ in a humidified incubator. The cells were harvested, resuspended, placed into a 2-ml tube and immediately used for the experiment at a concentration of $10^{6} \mathrm{ml}$ following reaching $80 \%$ confluence.

Ultrasound apparatus and ultrasound microbubbles. The experimental setup for ultrasound treatment is summarized in Fig. 1. The ultrasound apparatus (model, DM-40; Shanghai Acoustics Laboratory, Chinese Academy of Sciences) was used. The experimental apparatus mainly consisted of two parts; an ultrasonic generator and a transducer. The ultrasonic transducer units operating at a frequency of $45 \mathrm{kHz}$ [effective radiating area (ERA), $38.04 \mathrm{~cm}^{2}$ ] were positioned sidewise in the bottom of the water bath. The transducer was connected to an ultrasonic generator, which operated at a continuously adjustable frequency and its maximum output electric power was $\sim 50 \mathrm{~W}$. Also, the output electric power was monitored by a digital electric power analyzer (model, PPA2500; Newtons4th Ltd., Loughborough, UK). By adjusting the electric power, the ultrasound intensity was set. A needle-type hydrophone (model, ZS-1000; Yunchuan Electrical, Wuxi, China), positioned in the tube and connected to a digital oscilloscope (model, TDS 1024B; Tektronix, Beaverton, OR, USA), was used to examine the operating condition.

SonoVue ${ }^{\mathrm{TM}}$ microbubble echo-contrast agent (Bracco SpA, Milan, Italy) was used in combination with ultrasound treatment. The SonoVue ${ }^{\mathrm{TM}}$ agent (Bracco $\mathrm{SpA}$ ) used was a lipid-shelled ultrasound contrast agent composed of microbubbles that were filled with sulfur hexafluoride gas. The microbubbles were 2.5-6.0 $\mu \mathrm{m}$ in diameter. Prior to use, SonoVue ${ }^{\mathrm{TM}}$ was reconstituted in $5 \mathrm{ml}$ of phosphate-buffered saline (PBS) to a concentration of $2-5 \times 10^{8}$ microbubbles $/ \mathrm{ml}$.

Ultrasound treatment combined with microbubbles. The A7r5 cells were divided into different groups according to the different dosage of the SonoVue ${ }^{\mathrm{TM}}$ agent and the duration of ultrasound irradiation. Different volumes of SonoVue ${ }^{\mathrm{TM}}$ agent $(0,14,28,42$ and $56 \mu \mathrm{l})$ were added into a 2 -ml tube of A7r5 cells, respectively. Therefore, the concentration of microbubbles was $0,14-35 \times 10^{5}, 28-70 \times 10^{5}, 42-105 \times 10^{5}$ and $56-140 \times 10^{5}$ microbubbles/ml, accordingly. Different durations of ultrasound irradiation were applied as follows; 0, 10, 20 and $30 \mathrm{sec}$. The control group received neither treatment of ultrasound irradiation nor SonoVue ${ }^{\mathrm{TM}}$. All treatment groups were analyzed three times.

Cell viability. The cell viability was assessed by the trypan blue exclusion test immediately following the treatments. The trypan blue dye exclusion test was performed immediately following treatment by mixing $100 \mu \mathrm{l}$ of each cell suspension with an equal amount of $0.3 \%$ trypan blue solution (Sigma-Aldrich, St. Louis, MO, USA) in PBS (15). Following incubation at room temperature for $5 \mathrm{~min}$, the numbers of stained and unstained cells were counted using a hemocytometer to estimate the number of intact non-viable and viable cells, respectively. The count prior to exposure was considered to be the $100 \%$ intact count and the decrease in the number of intact cells following sonication was considered to be due to cell lysis (16).

Measurement of cell proliferation. A7r5 adherent cells were treated with $0.25 \%$ trypsin, suspended and harvested by centrifugation. After resuspending cells at $1 \times 10^{6} \mathrm{ml}$, serial dilutions of the cells in culture medium were prepared from $1 \times 10^{6}$ to $1 \times 10^{3}$ cells $/ \mathrm{ml}$. A total of $100 \mu \mathrm{l}$ of the dilutions were added into the wells of a microtiter plate in triplicate. Three control wells of medium alone were included to provide the blanks for absorbance readings. The cells under appropriate conditions were incubated for $24 \mathrm{~h}$. A total of $10 \mu \mathrm{l} \mathrm{MTT}$ reagent (Cell Proliferation Assay kit; American Type Culture Collection, Manassas, VA, USA) was added into each well, including the controls, following incubation for another $4 \mathrm{~h}$. When the purple precipitate was clearly visible under the microscope, $100 \mu \mathrm{l}$ of detergent reagent was added into all of the wells, including the controls. The plate was covered and stored in the dark for $4 \mathrm{~h}$ at room temperature. The absorbance in each well, including the blanks, was measured at $570 \mathrm{~nm}$ in a microtiter plate reader (Thermo Mustiskan Mk3; Thermo Life Sciences, Basingstoke, UK). 


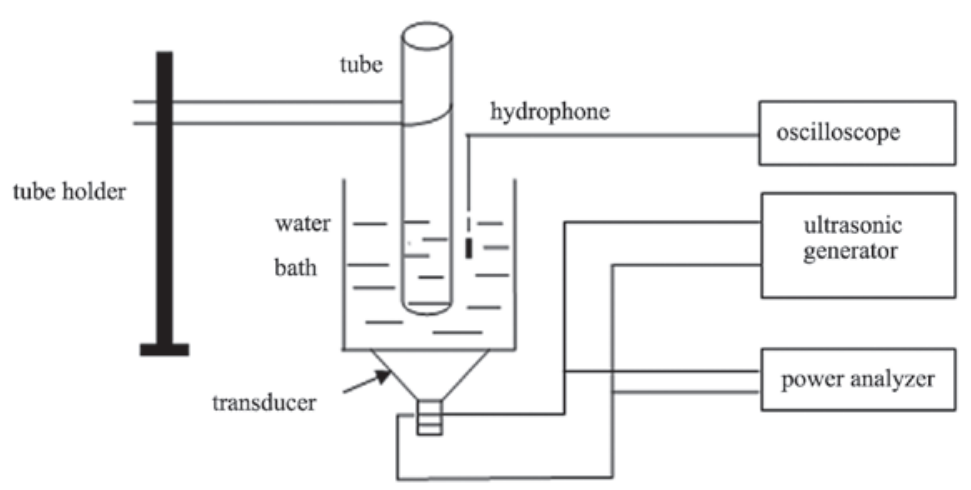

Figure 1. Experimental setup for the ultrasound treatment.

Cell apoptosis. The Annexin-V-fluorescein isothiocyanate (FITC) kit was purchased from Nanjing KeyGen Biotech. Co., Ltd. (Nanjing, China). Propidium iodide (PI) was purchased from Sigma-Aldrich. After treated as described above, A7r5 cells were resuspended in binding buffer $\left(1 \times 10^{6}\right.$ cells $\left./ \mathrm{ml}\right)$. Then, Annexin V-FITC and PI double-staining were performed according to manufacturer's instructions. Cell apoptosis was detected by flow cytometric analysis performed at $488 \mathrm{~nm}$ using a FACSCalibur flow cytometer (Becton-Dickinson, Franklin Lakes, NJ, USA).

Western blot analysis. B-cell lymphoma 2 (Bcl-2) and $\mathrm{Bcl}-2$-associated $\mathrm{X}$ (Bax) protein expression levels were measured using western blotting. Following 24 h, the treated and untreated cells were harvested and lysed, and the supernatants were separated from the cell debris by centrifugation at $13,400 \mathrm{xg}$ for $15 \mathrm{~min}$ at $4^{\circ} \mathrm{C}$. Aliquots containing $30 \mu \mathrm{g}$ of total protein were separated by SDS-PAGE and transferred onto nitrocellulose membranes. The membranes were probed with primary rabbit monoclonal antibodies against Bcl-2 (Beyotime Institute of Biotechnology, Shanghai, China) and Bax (Abnova, Walnut, CA, USA) at $4^{\circ} \mathrm{C}$ overnight. The membranes were subsequently probed with a goat anti-rabbit secondary antibody conjugated with horseradish peroxidase (Santa Cruz Biotechnology, Inc., Santa Cruz, CA, USA) and visualized using an enhanced chemiluminescence (ECL) system (Cell Signaling Technology, Danvers, MA, USA). Protein band densities were quantified using Bio-Rad Quantity One software (Bio-Rad Laboratories, Hercules, CA, USA).

Detection of cavitation. The acoustic cavitations were detected according to the method of Hauptmann et al (17). Hydrophones were placed into tubes which were filled with A7r5 cells treated with ultrasound (US group) or ultrasound combined with microbubbles (US+MB group), and the change of acoustic fields was detected over time. The data were fourier transformed to obtain the fundamental frequency of acoustic fields and the amplitude of the 2nd, 3rd and 4th harmonic acoustic pressure. The amplitude of fundamental frequency was set as the standard to normalize the data, where the normalized data represented the acoustic cavitation according to the method of Hauptmann et al (17). The experiments were repeated four times.

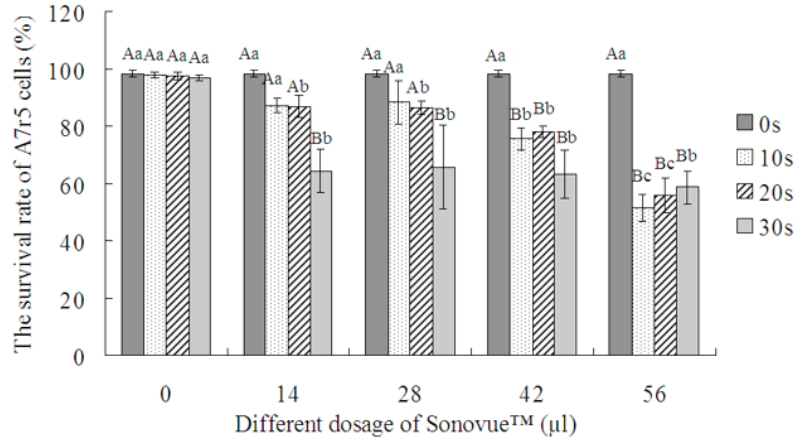

Figure 2. Survival rate (\%) of A7r5 cells following different treatments (mean \pm standard deviation, $n=3$ ). Capital letters indicate significant differences between values for the same dosage of SonoVue ${ }^{\mathrm{TM}}$, and lower case letters indicate significant differences between values for the same time of irradiation.

Statistical analysis. All data are expressed as the mean \pm standard deviation. All of the presented experiments were performed three times. Statistical analysis was performed using SPSS 16.0 (SPSS, Inc., Chicago, IL, USA). P $<0.05$ was considered to indicate a statistically significant difference between values.

\section{Results}

Cell viability. The survival rates of A7r5 cells treated with different concentrations of microbubbles and ultrasound irradiation for variable durations were examined by trypan blue staining (Fig. 2). The average survival rate of the control group was $98.33 \pm 1.15 \%$. With irradiation treatment for 10 or $20 \mathrm{sec}$, the survival rate of A7r5 cells was not significantly changed when the dosage of SonoVue ${ }^{\mathrm{TM}}$ was $<28 \mu$ l, but it significantly decreased with the treatment of $42 \mu \mathrm{l} \mathrm{SonoVue}{ }^{\mathrm{TM}}$. There was a significant difference between the survival rate of A7r5 cells treated with $42 \mu 1$ SonoVue ${ }^{\mathrm{TM}}$ and that of cells treated with $56 \mu \mathrm{l}$ SonoVue ${ }^{\mathrm{TM}}$. With the ultrasound irradiation treatment duration of $30 \mathrm{sec}$, the survival rate of A7r5 cells without SonoVue ${ }^{\mathrm{TM}}$ treatment was significantly higher than that of cells treated with different doses of SonoVue ${ }^{\mathrm{TM}}$. When the SonoVue ${ }^{\mathrm{TM}}$ dosage was $<28 \mu 1$, the survival rate of A7r5 cells treated with $10 \mathrm{sec}$ irradiation was not significantly different from that of cells treated with $20 \mathrm{sec}$ irradiation, but significantly higher than that of cells treated with $30 \mathrm{sec}$ irradiation. With the treatment with $42 \mu 1$ SonoVue ${ }^{\mathrm{TM}}$, the survival rates of A7r5 cells decreased with 
A

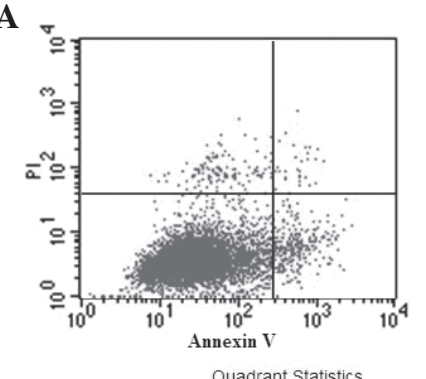

Quadrant Statistics

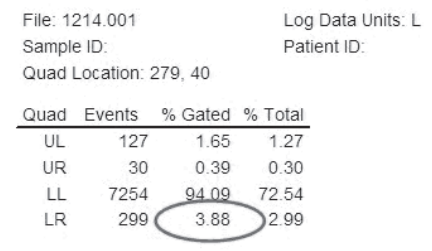

B
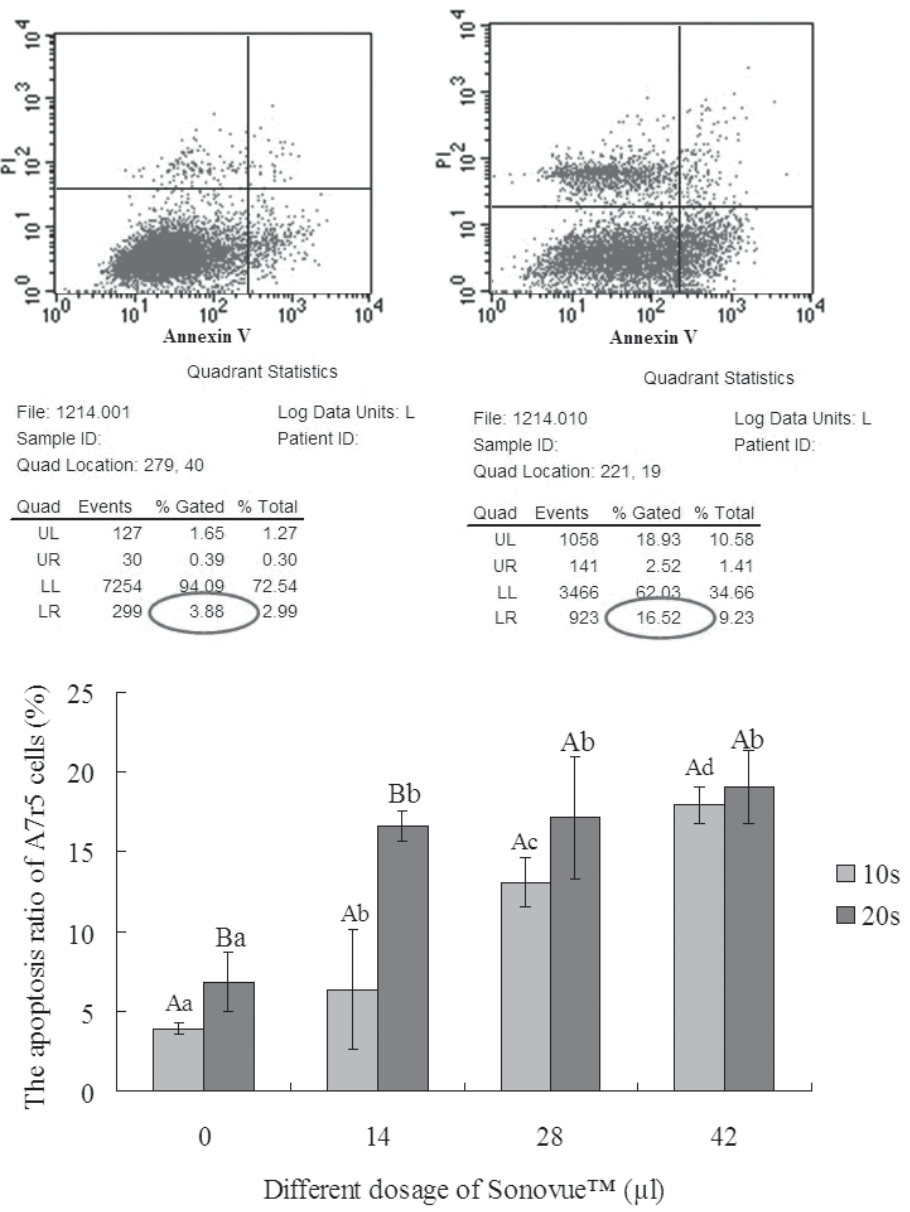

Figure 3. Apoptosis of A7r5 cells following different treatments. A) Flow cytometric analysis of apoptosis of cells treated with no (left) and 20 sec (right) irradiation combined with $14 \mu \mathrm{l} \mathrm{SonoVue}{ }^{\mathrm{TM}}$. B) Apoptotic rate (\%) of A7r5 cells. Different capital letters represent a significant difference between values for the same concentration of SonoVue ${ }^{\mathrm{TM}}$ and lowercase letters represent significant differences between values for the same time of irradiation.

increased irradiation time; however, there was no significant difference between the survival rate of $\mathrm{A} 7 \mathrm{r} 5$ cells treated with $10 \mathrm{sec}$ irradiation and that of cells treated with $20 \mathrm{sec}$ irradiation. Following treatment with $56 \mu \mathrm{l}$ SonoVue ${ }^{\mathrm{TM}}$, the survival rate of A7r5 cells without ultrasound treatment (treated for $0 \mathrm{sec}$ ) was significantly higher than that of cells that had received the other treatments. Trypan staining indicated that $30 \mathrm{sec}$ irradiation combined with different concentrations of microbubbles, or $56 \mu 1$ SonoVue ${ }^{\mathrm{TM}}$ combined with 10 or $20 \mathrm{sec}$ irradiation, both immediately induced marked cell death, which demonstrated the induction of damage to the A7r5 cells.

Cell apoptosis. Flow cytometric analysis was used to determine the apoptosis of the eight selected groups of cells treated for 10 or $20 \mathrm{sec}$ irradiation combined with different concentrations of SonoVue ${ }^{\mathrm{TM}}(0,14,28$ and $42 \mu \mathrm{l})$. Flow cytometry dot plots of cells stained with Annexin V/PI were used to determine the apoptotic rates of cells treated with no (left) and $20 \mathrm{sec}$ (right) irradiation combined with $14 \mu \mathrm{l} \mathrm{SonoVue}{ }^{\mathrm{TM}}$ (Fig. 3A). The apoptotic rates (\%) of $\mathrm{A} 7 \mathrm{r} 5$ cells treated with irradiation (10 and $20 \mathrm{sec})$ combined with different concentrations of SonoVue ${ }^{\mathrm{TM}}(0$, 14, 28 and $42 \mu \mathrm{l}$ ) are shown in Fig. 3B. Following treatment with $10 \mathrm{sec}$ ultrasound irradiation, the apoptotic rates of A7r5 cells increased with increasing microbubble concentration. There were significant differences in apoptotic rates of A7r5 cells

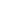

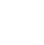

(2) 
A

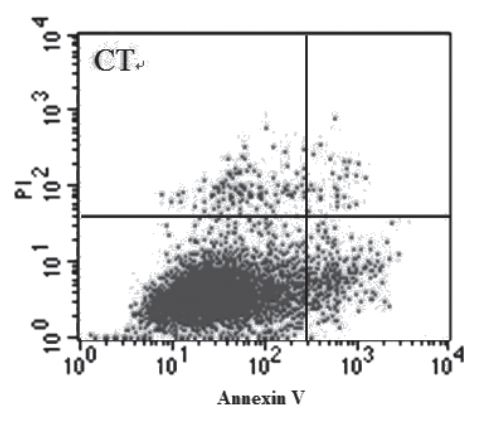

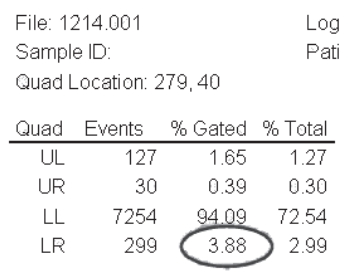

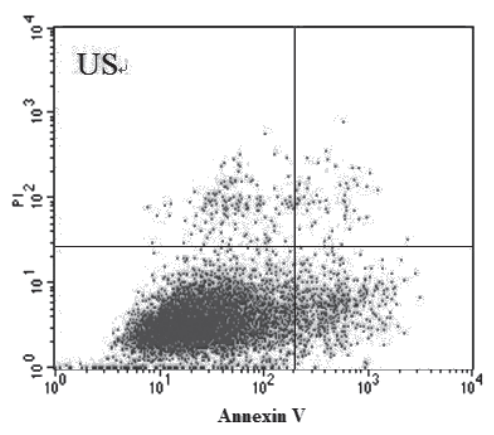

Quadrant Statistics

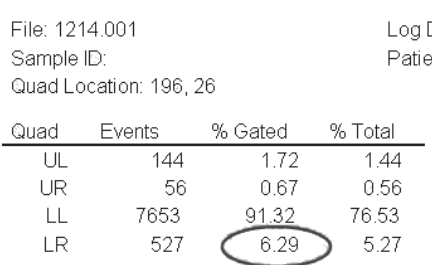

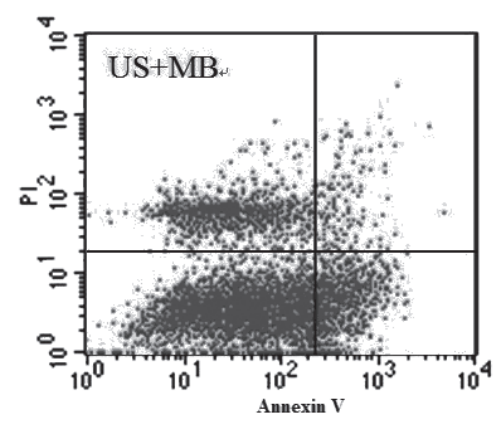

Quadrant Statistics

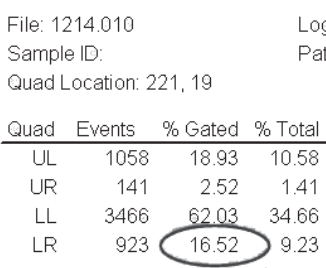

B

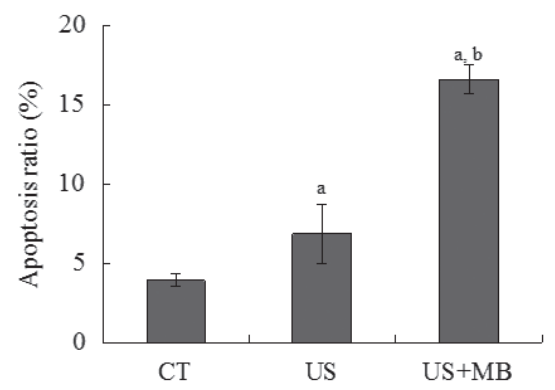

Figure 5. Apoptosis of A7r5 cells in the CT, US and US+MB groups at 24 h following treatment examined using flow cytometry. (A) Flow cytometric analysis of apoptosis in CT, US and US+MB groups. (B) The apoptotic rate in the groups. CT, control group; US, 20 sec ultrasound irradiation; US+MB, 20 sec ultrasound irradiation combined with $14 \mu 1$ microbubbles. ${ }^{\mathrm{a}} \mathrm{P}<0.01 \mathrm{vs}$. CT group; ${ }^{\mathrm{b}} \mathrm{P}<0.001$ vs. US group.

A

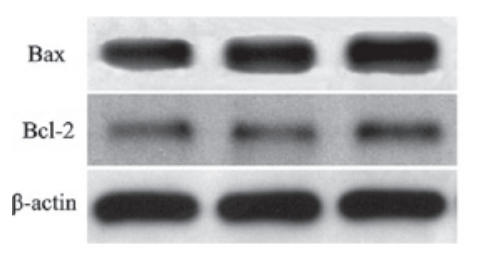

B

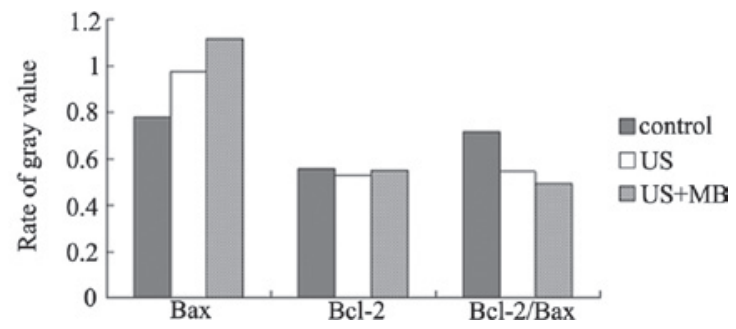

Figure 6. Expression of Bcl-2, Bax and Bcl-2/Bax in the CT, US and US+MB groups using western blotting analysis. CT, control group; US, 20 sec ultrasound irradiation; US+MB, $20 \mathrm{sec}$ ultrasound irradiation combined with $14 \mu 1$ microbubbles; Bcl-2, B-cell lymphoma 2; Bax, Bcl-2-associated X.

selected for the US+MB group for the characteristics of reduced necrosis, increased apoptotic rate and lowest microbubble dose.

The MTT assay revealed that the proliferation of A7r5 cells was inhibited in the US and US+MB groups compared with the control group. This suppression was greater in the US+MB than in the US group. However, these differences were not statistically significant (Fig. 4).

Apoptosis following treatment with ultrasound with or without microbubbles. Compared with the control group, the US treatment group demonstrated marginal cell apoptosis, and the US+MB treatment group exhibited significant levels of apoptosis. Accordingly, apoptosis was induced by both ultrasound irradiation alone and ultrasound irradiation combined with microbubbles. The use of microbubbles combined with ultrasound, however, was able to induce a significantly higher degree of apoptosis in the A7r5 cells (Fig. 5A and B).

Western blot analysis. Western blot analysis (Fig. 6) demonstrated that the expression of Bax in the US or US+MB groups was significantly higher than that in the control group. There was no significant difference in Bcl-2 expression among the three groups. This resulted in a certain decrease in the Bcl-2Bax ratio. 


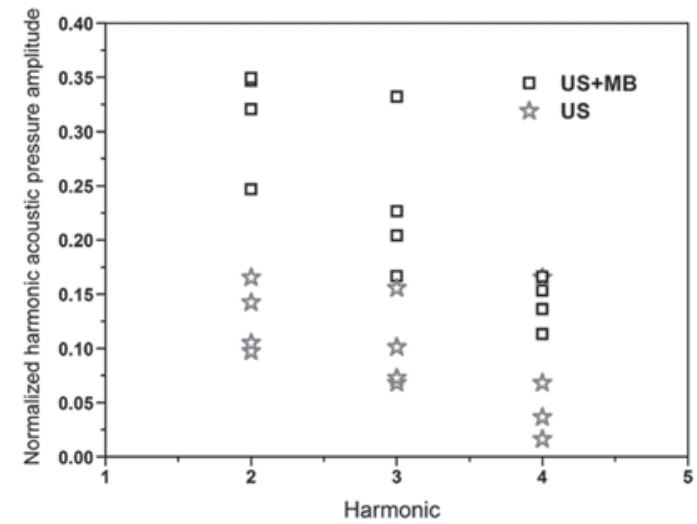

Figure 7. Normalized amplitude from second, third and fourth harmonic acoustic pressure. CT, control group; US, $20 \mathrm{sec}$ ultrasound irradiation; US+MB, $20 \mathrm{sec}$ ultrasound irradiation combined with $14 \mu 1$ microbubbles.

Detection of cavitation. The normalized harmonic acoustic pressure amplitudes of the US and US+MB groups were detected to represent the cavitation of the cells according to the method by Hauptmann et al (17)(Fig. 7). The results revealed that the harmonic acoustic pressure amplitude in the US+MB group was significantly larger than that in the US group, which suggested that the US+MB group exhibited an intense non-linear effect. Therefore, it was concluded that the cavitation intensity in the US+MB group was more intensive than that in the US group, and that the cavitation significantly affected the apoptosis of A7r5 cells, which may result from the intensive influence of jet and shock waves produced by expanding and collapsing from the cavitation nucleus.

\section{Discussion}

Ultrasound, as a kind of non-ionizing energy, has a number of mechanical, thermodynamical and chemical biological effects. The biophysical modes of ultrasound are divided into three classes of effect: Thermal, cavitational and mechanical (18). Numerous studies have demonstrated that the effects of ultrasound combined with microbubbles on treating a number of diseases are mainly attributed to the cavitations of microbubbles. Cells are damaged by the shock wave and high-speed jets, which are triggered by cavitations. During the cavitations, high-velocity gradient and viscosity stress existing on the bubble surface produces outgoing micro jets which induces a number of biological effects on the surrounding cells and biological macromolecules (19). More severe contraction of the cavitation bubble is often accompanied by shock waves, high-speed micro-jets, sonoluminescence, sonochemistry, etc. Cellular components proximal to the center of the cavitations will suffer severe damage and destruction. Ultrasound-stimulated microbubbles are able to induce significant bioeffect-associated changes in the gene expression and affect ceramide signaling pathways leading to apoptosis (9). During the process of irradiation with ultrasound, if the parameters are not selected appropriately, the cells may experience mass mortality in a short period, which may lead to vasomotor ataxia and an inflammatory reaction, as well as attenuation of the middle layer of arterial walls, which may cause aneurysm. Therefore, examining the effectiveness of ultrasound treatment and identifying a relatively reasonable and safe treatment, which induces VSMC apoptosis and inhibits VSMC proliferation without large amounts of necrosis, is highly important.

In the present study, SonoVue ${ }^{\mathrm{TM}}$ microbubble echo-contrast agent was used in combination with ultrasound treatment. The agent used was a lipid-shelled ultrasound contrast agent composed of microbubbles filled with sulfur hexafluoride gas. The microbubbles were 2.5-6.0 $\mu \mathrm{m}$ in diameter. In the present study, different concentrations of SonoVue ${ }^{\mathrm{TM}}$ microbubbles were used combined with $45 \mathrm{kHz} 0.3 \mathrm{Wcm}^{2}$ ultrasound to examine the effects on apoptosis of VSMCs. Clinically, ultrasound is described as being of low, medium or high intensity at values of $0-0.5,0.5-3$ and $>3 \mathrm{Wcm}^{2}$, respectively (20). As a result of the high energies involved in ultrasound treatment, cell lysis is the major result and it is possible that other effects on the surviving cells are masked $(21,22)$. Different settings of frequency and energy density in ultrasonic treatment procedures lead to different impacts on cell proliferation, morphology and differentiation, and may be used to stimulate or inhibit the growth of individual cell types (23). In previous studies, ultrasound of $1 \mathrm{MHz}$ or $>1 \mathrm{MHz}$ was usually used by a combination of microbubbles, which induced apoptosis $(20,24)$. Ultrasound frequencies markedly $<1 \mathrm{MHz}$, in particular $\sim 45 \mathrm{kHz}$, have not been widely studied (11).

Optison and YM454 are effective in augmenting US-induced cell death, but not Levovist. Levovist minimally enhanced the US-induced apoptosis at $1.0 \mathrm{Wcm}^{2}$ while Optison and YM454 showed effects at 2.0 and $4.0 \mathrm{Wcm}^{2}(18)$. Cavitation has a role in the augmented effects and this inertial cavitation appears necessary for Optison and YM454 to effect their actions. In the present study, SonoVue ${ }^{\mathrm{TM}}$, as a type of microbubbles, had a marked cavitational effect on inducing VSMC apoptosis when it was used in combination with low frequency low energy ultrasound. A7r5 cells were treated by a combination of varied ultrasonic irradiation durations and different microbubble concentrations. The results demonstrated that cell death and apoptosis were associated with both irradiation duration and microbubble concentration. The survival rates of A7r5 cells were significantly decreased when the irradiation time exceeded $30 \mathrm{sec}$, with the combination treatment of $14 \mu 1$ microbubbles. A significant decrease in the survival rate of the cells was also identified when cells were treated with $56 \mu$ l SonoVue ${ }^{\mathrm{TM}}$ combined with 10 or $20 \mathrm{sec}$ irradiation. These results suggested that excess ultrasonic irradiation time with microbubbles or excess microbubble concentration with ultrasound irradiation may induce immediate cell death.

Furthermore, the present study further examined the effect of different combinations of irradiation time and microbubble concentrations on the apoptosis of A7r5 cells below the excess conditions (30 sec irradiation and $56 \mu$ l SonoVue ${ }^{\mathrm{TM}}$ ). With the treatment of $10 \mathrm{sec}$ irradiation, the apoptotic rate was enhanced with increasing microbubble concentration. Of note, when the microbubble dosage was increased from 14 to $42 \mu \mathrm{l}$, there was no significant increase in cell apoptosis.

In the present study, the combination of $20 \mathrm{sec}$ ultrasound irradiation combined with $14 \mu 1$ microbubbles was an effective method to induce A7r5 cell apoptosis without triggering marked necrosis. The apoptotic rates of the group treated 
with $20 \mathrm{sec}$ ultrasound irradiation combined with $14 \mu \mathrm{l}$ microbubbles, and of the group treated with $20 \mathrm{sec}$ ultrasound irradiation were significantly higher than those of the control group $(\mathrm{P}<0.01)$, and the apoptotic rate of this combination treated group was significantly higher than that of the $20 \mathrm{sec}$ ultrasound irradiation group $(\mathrm{P}<0.001)$. Furthermore, the cavitation intensity of the combination treated group was also significantly higher than that of the $20 \mathrm{sec}$ ultrasound irradiation group. These results suggested that addition of SonoVue ${ }^{\mathrm{TM}}$ increased the amount of cavitation nuclei, and the cavitational effect was more intense than before, and therefore had a stronger bioeffect on the cells. Therefore, $45 \mathrm{kHz}$ of ultrasonic waves with $0.3 \mathrm{Wcm}^{2}$ of intensity may induce apoptosis mainly through cavitational effects.

Apoptosis is the process of programmed cell death and is generally characterized by distinct morphological characteristics and energy-dependent biochemical mechanisms (25). The apoptotic process appears to be regulated by a number of factors, including members of the Bcl-2 family of proteins. Bcl-2 family members determine cell death and survival by controlling mitochondrial membrane ion permeability, the release of cytochrome $\mathrm{c}$ and the subsequent activation of caspase (caspase 3 and caspase 9) executor functions (26). The Bcl-2 family of proteins consists of pro-apoptotic (Bax, $\mathrm{Bcl}$ extra small, Bcl-2 homologous killer and Bcl-2-associated death promoter) and anti-apoptotic ( $\mathrm{Bcl}-2, \mathrm{Bcl}$ extra large and Bcl-2-like protein 2) proteins (27). VSMCs have an important role in PTA. However, there are few studies examining the effect of ultrasound on the apoptosis and apoptosis-associated genes of VSMCs. The present study identified an increased Bax expression and decreased $\mathrm{Bcl}-2 \mathrm{Bax}$ ratio in the US+MB group compared with those in the control and US groups, demonstrating the possible mechanism by which a combination treatment of ultrasound and microbubbles induced apoptosis in the cells.

In conclusion, low frequency low energy ultrasound combined with microbubbles may induce apoptosis of VSMCs, mainly through cavitation, and the regulation of Bcl-2 and Bax expression. These findings demonstrated that appropriate selection of ultrasound conditions combined with microbubbles may be a considerable strategy for treating restenosis of blood vessels following PTA and stenting.

\section{Acknowledgements}

The present study was supported by Shanghai Pujiang Project of China (grant no. 09PJ1408800), the Major Infrastructure Projects of Shanghai Science and Technology (grant no. 10JC1412600), the Academic Leaders Training Program of Pudong Health Bureau of Shanghai (grant no. PWRd201302), and the National Natural Science Foundation of China (grant no. 81271597).

\section{References}

1. Reimers B, Moussa I, Akiyama T, et al: Long-term clinical follow-up after successful repeat percutaneous intervention for stent restenosis. J Am Coll Cardiol 30: 186-192, 1997.

2. Kaufman GE, Miller MW, Griffiths TD, et al: Lysis and viability of cultured mammalian cells exposed to $1 \mathrm{MHz}$ ultrasound. Ultrasound Med Biol 3: 21-25, 1977.
3. Ellwart JW, Brettel H and Kober LO: Cell membrane damage by ultrasound at different cell concentrations. Ultrasound Med Biol 14: 43-50, 1988.

4. Fahnestock M, Rimer VG, Yamawaki RM, et al: Effects of ultrasound exposure in vitro on neuroblastoma cell membranes. Ultrasound Med Biol 15: 133-144, 1989.

5. Clarke PR and Hill CR: Physical and chemical aspects of ultrasonic disruption of cells. J Acoust Soc Am 47: 649-653, 1970.

6. Carstensen EL, Kelly P, Church CC, et al: Lysis of erythrocytes by exposure to $\mathrm{CW}$ ultrasound. Ultrasound Med Biol 19: 147-165, 1993.

7. Miller DL and Williams AR: Bubble cycling as the explanation of the promotion of ultrasound in a rotating tube exposure system. Ultrasound Med Biol 15: 641-648, 1989.

8. Thacker J: An approach to the mechanism of killing of cells in suspension by ultrasound. Biochim. Biophys Acta 304: 240-248, 1973.

9. Al-Mahrouki AA, Karshafian R, Giles A and Czarnota GJ: Bioeffects of ultrasound-stimulated microbubbles on endothelial cells: gene expression changes associated with radiation enhancement in vitro. Ultrasound Med Biol 38: 1958-1969, 2012.

10. Feril LB Jr, Kondo T, Zhao QL, et al: Enhancement of ultrasound-induced apoptosis and cell lysis by echo-contrast agents. Ultrasound Med Biol 29: 331-337, 2003.

11. Zhang B, Zhou HS, Cheng Q, Lei L, Hu B: Low-frequency ultrasound induces apoptosis of rat aortic smooth muscle cells (A7r5) via the intrinsic apoptotic pathway. Genet Mol Res 13: 3143-3153, 2014

12. Rokhina EV, Lens P and Virkutyte J: Low-frequency ultrasound in biotechnology: state of the art. Trends Biotechnol 27: 298-306, 2009.

13. Wang Y, Hu B, Diao X and Zhang J: Antitumor effect of microbubbles enhanced by low frequency ultrasound cavitation on prostate carcinoma xenografts in nude mice. Exp Ther Med 3: 187-191, 2012.

14. Man J, Shelton RM, Cooper PR and Scheven BA: Low-intensity low-frequency ultrasound promotes proliferation and differentiation of odontoblast-like cells. J Endod 38: 608-613, 2012.

15. Lagneaux L, de Meulenaer EC, Delforge A, et al: Ultrasonic low-energy treatment: a novel approach to induce apoptosis in human leukemic cells. Exp Hematol 30: 1293-1301, 2002.

16. Furusawa Y, Zhao QL, Hassan MA, et al: Ultrasound-induced apoptosis in the presence of Sonazoid and associated alterations in gene expression levels: a possible therapeutic application. Cancer Lett 288: 107-115, 2010.

17. Hauptmann M, Brems S, Struyf H, et al: Time-resolved monitoring of cavitation activity in megasonic cleaning systems. Rev Sci Instrum 83: 034904, 2012.

18. Sicklick JK, Li YX, Jayaraman A, et al: Dysregulation of the Hedgehog pathway in human hepatocarcinogenesis. Carcinogenesis 27: 748-757, 2006.

19. Gormley G and Wu J: Observation of acoustic streaming near Albunex spheres. J Acoust Soc Am 104: 3115-3118, 1998.

20. Jackson JK, Pirmoradi FN, Wan CP, et al: Increased accumulation of paclitaxel and doxorubicin in proliferating capillary cells and prostate cancer cells following ultrasound exposure. Ultrasonics 51: 932-939, 2011.

21. Kawai $\mathrm{N}$ and Iino $\mathrm{M}$ : Molecular damage to membrane proteins induced by ultrasound. Ultrasound Med Biol 29: 609-614, 2003.

22. Marentis TC, Kusler B, Yaralioglu GG, et al: Microfluidic sonicator for real-time disruption of eukaryotic cells and bacterial spores for DNA analysis. Ultrasound Med Biol 31: $1265-1277,2005$.

23. Hutcheson JD, Schlicher RK, Hicks HK and Prausnitz MR: Saving cells from ultrasound-induced apoptosis: quantification of cell death and uptake following sonication and effects of targeted calcium chelation. Ultrasound Med Biol 36: 1008-1021, 2010.

24. Tabuchi Y, Takasaki I, Zhao QL, et al: Genetic networks responsive to low-intensity pulsed ultrasound in human lymphoma U937 cells. Cancer Lett 270: 286-294, 2008.

25. Coffin AB, Williamson KL, Mamiya A, et al: Profiling drug-induced cell death pathways in the zebrafish lateral line. Apoptosis 18: 393-408, 2013.

26. Barazzone $\mathrm{C}$ and White $\mathrm{CW}$ : Mechanisms of cell injury and death in hyperoxia: role of cytokines and $\mathrm{Bcl}-2$ family proteins. Am J Respir Cell Mol Biol 22: 517-519, 2000.

27. Adams JM and Cory S: The Bcl-2 protein family: arbiters of cell survival. Science 281: 1322-1326, 1998. 